\title{
Nereida Congost-Maestre* \\ La Validación Lingüística en los Principales \\ Cuestionarios de Salud y Calidad de Vida
}

DOI 10.1515/les-2015-0014

\begin{abstract}
The goals of this article are to summarize the problems and solutions found in translating seven Health-Related Quality of Life (HRQOL) questionnaires from English into Spanish which have used a common international protocol based on back-translation techniques. The methodology used is based on the linguistic validation model including both the linguistic and the sociopragmatic equivalence. Five questionnaires from seven have obtained good results, not so two of them. Considering linguistic questions, there were more problems than good solutions on the lexical-semantic level. With respect to the sociocultural questions, there were more solutions than problems. The Spanish translated questionnaires still present deficiencies to be corrected, so both linguistic and sociocultural questions have to be studied more carefully in order not to allow differences between the translated versions and the source questionnaires.
\end{abstract}

Schlagwörter: Traducción, retrotraducción, validación lingüística, adaptación cultural, calidad de vida relacionada con la salud, cuestionarios

\section{Introducción}

Los dos tipos más importantes de validación al hablar de comparabilidad transcultural son la lingüística y la psicométrica (Acquadro et al., 2008) aunque nosotros nos vamos a centrar sólo en la primera por ser nuestro ámbito de estudio. De este modo, nuestro propósito ha sido el de analizar y valorar hasta qué punto y en qué medida se ha alcanzado la validación lingüística en los principales cuestionarios de Calidad de Vida Relacionada con la Salud (CVRS), basándonos en estudios realizados sobre los instrumentos SF-36, COOP/WONCA, NPH o EQ-5D y a partir del modelo de adaptación lingüística y cultural propuesto por el comité científico del Medical Outcomes Trust para instrumentos adaptados (Scientific Advisory Committee of the Medical Outcomes Trust, 2002).

*Corresponding author: Nereida Congost-Maestre, Departamento de Estudios Ingleses, Facultad de Filosofía y Letras, Universidad de Alicante, España, E-Mail: nereida.congost@ua.es 
La mayoría de los cuestionarios genéricos de CVRS han sido desarrollados inicialmente en Estados Unidos y Gran Bretaña. Este hecho, unido a la particularidad de que su elaboración tenga un coste elevado y requiera un largo tiempo de preparación, obliga a adaptarlos a la nueva cultura, en nuestro caso la española, en una tarea que consiste en la traducción y adaptación cultural del cuestionario.

Se ha visto que el interés de los estudios comparativos culturales (transculturales e interculturales) no ha parado de crecer en las últimas tres décadas: un instrumento que haya mostrado buenas características métricas de calidad y que haya obtenido resultados interesantes en una cultura, se quiere probar en otras con el fin de examinar diferencias y similitudes culturales, lo cual requiere un esfuerzo mayor que el de realizar una simple traducción.

Así pues, la traducción constituye el primer paso para obtener una nueva versión de un cuestionario en un idioma diferente y es un elemento fundamental para conseguir una equivalencia con la versión original. Se puede considerar tan importante como el proceso original del desarrollo del instrumento.

El segundo paso consistiría en la adaptación del cuestionario cuando la cultura de destino lo requiera. El proceso de conformar un contenido a la visión particular de cada lengua (es decir, la traducción de una diferencia cultural) se conoce como "adaptación". Con este procedimiento, la traducción adquiere "viabilidad cultural" (Vázquez-Ayora, 1977). El traductor no sólo debe conocer perfectamente las lenguas implicadas en el proceso de traducción, sino también las culturas a las que estas pertenecen.

Hablamos, pues, de equivalencia lingüística propiamente dicha cuando nos referimos a la formulación de los conceptos del cuestionario de forma que las estructuras sintácticas y léxico-semánticas seleccionadas cumplan la misma función y posean el mismo significado, tanto para cada ítem como para las opciones de respuesta y otros aspectos del instrumento (Guyatt, 1993; Herdman et al., 1998; Valderas et al., 2005).

En cuanto a la equivalencia sociopragmática, es aquella que trata de las diferencias culturales entre los pueblos y de la necesidad de que haya una adaptación cultural del instrumento (Bullinger et al., 1998; Beaton et al., 2000).

Para alcanzar estos dos tipos de equivalencia, dentro del modelo de validación lingüística, conviene seguir un proceso cuidadoso de adaptación que ha sido objeto de sistematización repetida. Los investigadores han llegado, durante los últimos años, a un cierto consenso respecto a la metodología más adecuada para el proceso de traducción y adaptación intercultural del cuestionario original, que permita su aplicación a la nueva cultura, por lo que el procedimiento que más se utiliza en la actualidad, aunque no resuelva todos los problemas, es el de traducción - retrotraducción (Bullinger et al., 1998; Beaton et al., 2000; 
Badia et al., 2002, Valderas et al., 2005; Wild et al., 2005; Acquadro et al., 2012).

Este método consiste en que un grupo de traductores españoles, bilingües, que trabajan de forma independiente, realizan, al menos, dos traducciones del cuestionario original inglés al español. Traductores y autores elaboran una primera versión de común acuerdo. A continuación, un segundo grupo de traductores anglosajones, bilingües, que trabajan de forma independiente y que no conocen la versión original, toman el cuestionario traducido en español y lo vuelven a traducir al inglés. Traductores y autores elaboran una segunda versión consensuada. Este proceso se repite hasta que las dos versiones en inglés, la original y la retrotraducida, sean idénticas o muy similares.

Una vez obtenida la versión final del cuestionario, se realiza una prueba preliminar o estudio piloto en un grupo de individuos de similares características a las de la población a la que se pretende administrar el cuestionario para comprobar, mediante una entrevista cognitiva, que no hay ningún problema de comprensión en instrucciones, ítems y opciones de respuesta (Valderas et al., 2005 Acquadro et al., 2012).

\section{Métodos}

Hemos analizado siete cuestionarios genéricos de CVRS ampliamente aceptados en la literatura internacional, como hemos señalado en la Introducción, basándonos en estudios realizados sobre instrumentos como el SF-36, el COOP/WONCA, el NPH o el EQ-5D y a partir del modelo de adaptación cultural y lingüística propuesto por el comité científico del Medical Outcomes Trust para instrumentos adaptados (Scientific Advisory Committee of the Medical Outcomes Trust, 2002).

Los siete cuestionarios observados, previamente solicitado su permiso a los organismos correspondientes, son los que siguen:

1. Darmouth COOP Functional Health Assessment Charts/Wonca (COOP/WONCA) / Viñetas COOP/WONCA.

2. The European Quality of Life Scale (EQ-5D) / Cuestionario EuroQol-5D (EQ5D).

3. Nottingham Health Profile (NHP) / Perfil de Salud de Nottingham (PSN).

4. SF-36 Health Survey (SF-36v2) / Cuestionario de Salud SF-36v2.

5. Spitzer Quality of Life Index / Índice de Calidad de Vida de Spitzer.

6. Quality of Well-Being Scale - Self-Administered (QWB-SA) / Escala de Calidad del Bienestar-Autoadministrado (QWB-SA).

7. World Health Organization Quality of Life (WHOQOL-BREF) / Instrumento WHOQOL abreviado (WHOQOL-BREF). 
Las razones de dicha elección responden a los criterios siguientes:

- Todos están escritos en inglés y cuentan con una versión en castellano, a excepción de la Escala de Calidad del Bienestar-Autoadministrado (QWB-SA) que tiene únicamente una versión española (válida para España, Latinoamérica y población hispana en EEUU).

- Son cuestionarios canónicos estudiados en proyectos internacionales.

- Han seguido un protocolo común internacional basado en el método de traducción-retrotraducción, llevado a cabo por profesionales y probado en estudios piloto con pacientes.

- Han secundado el proceso de adaptación lingüística y cultural.

Nuestro sistema de trabajo ha consistido en: analizar el texto de la lengua de partida, analizar el texto de la lengua de llegada y comparar el análisis de ambos textos, comprobando en la traducción si se ha conseguido una validación lingüística y en qué medida.

Así mismo, las equivalencias que hemos considerado en nuestro modelo de validación lingüística son las que hemos señalado con anterioridad:

- la equivalencia lingüística, en los planos léxico-semántico, morfosintáctico y ortográfico de la lengua; $y$

- la equivalencia sociopragmática, en el plano conceptual, dirigida a la traducción de una diferencia ligada a la cultura en el contexto de la lengua de llegada.

Nuestro modelo de validación lingüística lo representamos del modo siguiente:

1. Equivalencia lingüística: cuestiones lingüísticas

1.1. Nivel oracional

(a) Plano léxico-semántico

(b) Plano morfosintáctico

(c) Plano ortográfico

1.2. Nivel supraoracional (plano textual)

2. Equivalencia sociopragmática: cuestiones socioculturales

2.1. Nivel de conocimiento de mundo (plano conceptual)

En la primera de las equivalencias, la lingüística, hemos analizado los problemas que surgen en la traducción en relación a las cuestiones lingüísticas, en las que hemos considerado los niveles oracional y supraoracional del lenguaje y en los que se ha estudiado, a su vez, los aspectos lingüísticos propiamente dichos así como la organización textual.

En el nivel oracional se tratarán las preferencias léxicas, sintácticas y ortográficas de cada lengua: 
(a) En el plano léxico-semántico se han analizado las palabras o expresiones que, a nuestro entender, se han traducido de manera equivocada, las modificaciones por sustitución, inclusión u omisión de un elemento semántico, los falsos amigos, las expresiones coloquiales, la repetición léxica, la redundancia léxica (y sintáctica), el grado de formalidad, el registro léxico diferente, los términos polisémicos, las oraciones largas, complejas o equívocas, las frases negativas, la gradación de las opciones de respuesta, la reducción léxica, etc. Además, se han considerado determinadas elecciones en la traducción, así como las traducciones poco afortunadas y las adaptaciones bien hechas.

(b) En el plano morfosintáctico se ha observado la concordancia gramatical de género y número, el modo y los tiempos verbales, los anglicismos sintácticos, los marcadores (artículos, conjunciones, preposiciones, adverbios, etc.), la voz pasiva, los adverbios acabados en -mente, los gerundios, el paralelismo gramatical, etc.

(c) En el plano ortográfico se ha considerado la puntuación de la frase, el uso de puntos suspensivos, la tilde o acento gráfico, los signos de interrogación, el empleo de mayúsculas, etc.

En el nivel supraoracional, por otra parte, se ha estudiado, en el plano textual, la organización textual del cuestionario: el nombre del cuestionario, los datos de cumplimentación (la fecha, por ejemplo), las instrucciones y el cuerpo propiamente dicho del cuestionario, es decir, las distintas secciones del instrumento (apartados, subapartados), las preguntas e ítems, el tipo de opciones de respuesta (dicotómicas, de opción múltiple, etc.); y otras preguntas opcionales, tales como el apartado de características demográficas y socioeconómicas del participante (que incluye sexo, edad, educación, ocupación, ingresos, etc.), experiencia en enfermedades (propia, en familiares y en otras personas), dificultad para cumplimentar el cuestionario y/o experiencia que se tiene en completar cuestionarios similares. Para finalizar, nos hemos fijado, además, en las fórmulas corteses (las palabras por favor y gracias) en ambas versiones y, además, en las marcas de tratamiento de tú o de usted o de adecuación de género (el uso del masculino y femenino en la misma cuestión) en nuestra versión.

La equivalencia lingüística se necesita para que el cuestionario traducido sea tan válido como el original. No obstante, hay una falta de consenso notable y una enorme confusión terminológica en el campo de las equivalencias.

Así pues, aquí se ha contemplado todo aquello que diversos autores han denominado, en una confusa gama de etiquetas:

(1) equivalencia semántica, designada como semantic equivalence (Hunt, 1986; Esteve-Vives et al., 1991; Hunt y Mckenna, 1992; Guillemin et al., 1993; Sartorius y Kuyken, 1994; Guarnaccia, 1996; Gaite et al., 1997; Herdman et al., 1998; Cella et al., 1998; Knudsen et al., 2000; Behling y Law, 2000; Skevington, 
2002; Hilton y Skrutkowski, 2002; Massoubre et al., 2002; Beck et al., 2003; Matías-Carrelo et al., 2003); functional equivalence (Bullinger, 1995) o conceptual equivalence (Leplège y Verdier, 1995; Anderson et al., 1996);

(2) equivalencia idiomática (idiomatic equivalence) (Sechrest et al., 1972; Guillemin et al., 1993; Beaton et al., 2000; Massoubre et al., 2002; Falcão et al., 2003); o

(3) equivalencia de las características "técnicas" de la lengua (technical equivalence), es decir, la morfosintaxis, la ortografía, los signos de puntuación, etc. (Alonso y Antó, 1990; Esteve-Vives et al., 1991; Sartorius y Kuyken, 1994; Gaite et al., 1997; Knudsen et al., 2000, Corless et al., 2001; Massoubre et al., 2002; Matías-Carrelo et al., 2003; Valderas et al., 2005).

Por lo que respecta a la equivalencia sociopragmática, hemos abordado cuestiones sociales y culturales que intervienen en todo proceso de comunicación humana. En este nivel de conocimiento del mundo, en el plano conceptual, es donde el traductor debe poseer un saber pragmático exhaustivo de la cultura de las comunidades lingüisticas a las que sirve de puente, para salvar cualquier diferencia que pueda surgir en la adaptación de los cuestionarios. La falta de simetría cultural o anisomorfismo parte de la idea de que cada lengua tiene su propio genio y personalidad, lo cual implica una diferencia cultural derivada de la historia, las instituciones y los hábitos que les son propios a cada comunidad y que son aspectos emic, es decir, conceptos específicos de una cultura determinada.

Por todo ello, hemos estudiado en este punto las traducciones de las diferencias culturales, adecuadas o desafortunadas, dependiendo de si se han tenido o no en cuenta los contextos situacional y cultural en el que se enmarcan los cuestionarios de salud y calidad de vida traducidos.

En esta segunda equivalencia, hemos incluido aquellos aspectos traductológicos de carácter sociopragmático y cultural, que diferentes autores han dado en llamar:

(1) equivalencia conceptual o funcional (conceptual equivalence, functional equivalence, conceptual [or functional] equivalence) (Alonso y Antó, 1990; EsteveVives et al., 1991; Hunt y Mckenna, 1992; Patrick et al., 1994; Sartorius y Kuyken, 1994; Gaite et al., 1997; Bullinger et al., 1998; Knudsen et al., 2000; Behling y Law, 2000; Jones et al., 2001; Massoubre et al., 2002; Skevington, 2002; Beck et al., 2003; Bowden et al., 2003);

(2) equivalencia de los ítems (item equivalence) (Herdman et al., 1998);

(3) equivalencia de contenido (content equivalence) (Guarnaccia, 1996; Cella et al., 1998; Hilton y Skrutkowski, 2002; Beck et al., 2003; Matías-Carrelo et al., 2003);

(4) equivalencia ligada a la experiencia (experiential equivalence, èquivalence liée à l'expèrience) (Sechrest et al., 1972; Guillemin et al., 1993; Beaton et al., 
2000; Massoubre et al., 2002); o (5) equivalencia normativa (normative equivalence) (Law, 2000).

\section{Resultados}

De los siete cuestionarios genéricos de CVRS seleccionados, la equivalencia lingüística y sociopragmática ha sido, en general, buena en cinco de ellos: SF36v2, COOP/WONCA, NHP, EQ-5D y Spitzer. Son instrumentos que han sido bien traducidos y adaptados, aunque con puntualizaciones. En dos de ellos, el WHOQOL-BREF y el QWB-SA, sin embargo, las equivalencias han sido menos afortunadas.

En este estudio, hemos detectado que en relación a las cuestiones lingüísticas en el nivel oracional hay más pérdidas que ganancias, sobre todo, en el plano léxico-semántico, seguido este de los planos morfosintáctico y ortográfico. En el nivel supraoracional, sin embargo, se ha ganado más que se ha perdido. La organización textual es clara y ordenada en su mayor parte y hay un empleo creciente de fórmulas de cortesía. Aún falla, no obstante, la adecuación de género a la hora de evitar formas lingüísticas sexistas.

Por lo que se refiere a las cuestiones socioculturales, hallamos tanto pérdidas como ganancias, pero con estas últimas en alza.

De los siete cuestionarios analizados, encontramos en las cuestiones lingüísticas:

- Redundancia léxica (y sintáctica) en seis: SF-36v2, COOP-WONCA, NHP, EQ5D, WHOQOL-BREF y QWB-SA.

- Grado de formalidad alto en cinco: SF-36v2, COOP-WONCA, Spitzer, WHOQOL-BREF y QWB-SA.

- Palabras mal traducidas en cinco: SF-36v2, NHP, EQ-5D, WHOQOL-BREF y QWB-SA.

- Uso del adjetivo moderado en cuatro: SF-36v2, COOP-WONCA, EQ-5D y WHOQOL-BREF.

- Oraciones largas con muchos conceptos en tres: COOP-WONCA, Spitzer y QWB-SA.

- Uso de adverbios formales acabados en - mente en cuatro: COOP-WONCA, NHP, Spitzer y WHOQOL-BREF.

- Puntuación insuficiente o inadecuada en los siete: SF-36v2, COOP-WONCA, NHP, EQ-5D, Spitzer, WHOQOL-BREF y QWB-SA.

- Organización textual satisfactoria en seis de los siete cuestionarios (en todos excepto en el QWB-SA); fórmulas de cortesía en los siete; adecuación de género sólo en uno (NHP), en otro no era necesario (COOP-WONCA) y en 
cinco no se ha tenido en cuenta (SF-36v2, EQ-5D, Spitzer, WHOQOL-BREF y QWB-SA).

En las cuestiones socioculturales, las adaptaciones de diferencias culturales se han llevado a cabo en su totalidad en dos cuestionarios (SF-36v2, NHP), en tres han sido adaptados algunos conceptos (COOP-WONCA, QWB-SA, Spitzer) y en dos no hay cuestiones socioculturales relevantes que haya que adaptar a nuestro entorno (EQ-5D, WHOQOL-BREF).

\section{Discusión}

Enumeraremos, pues, los instrumentos de mejor a peor, a la vista de los resultados obtenidos (véase análisis en el Anexo):

- SF-36v2: Es un cuestionario modelo, aunque debe revisar algunos aspectos lingüísticos, sobre todo en los planos léxico-semántico y ortográfico.

- COOP/WONCA: Tiene que revisar aspectos lingüísticos y ha de considerar una cuestión sociocultural.

- NHP: Tiene que mejorar aspectos lingüísticos.

- EQ-5D: Tiene que mejorar aspectos lingüísticos. Redacción pobre en castellano.

- SPITZER: Tiene que revisar y mejorar aspectos lingüísticos y ha de considerar dos cuestiones socioculturales.

- -WHOQOL-BREF: Plantea problemas léxicos y semánticos en enunciados, preguntas y opciones de respuestas. Redacción pobre en instrucciones e ítems.

- QWB-SA: Se recomienda una revisión completa del cuestionario en todos los aspectos:

- Plantea problemas léxicos, sintácticos y semánticos.

- Plantea problemas de registro léxico al haber una única versión para todos los países de habla hispana y, por tanto, de aplicabilidad inmediata.

- Tiene que revisar la organización textual y adaptar las diferencias culturales.

Estos resultados se pueden observar de forma gráfica en la siguiente tabla (ver Tabla 1): 
Table 1: Comparación de la equivalencia lingüística $(1.1,1.2)$ y sociopragmática (2.1) en siete instrumentos genéricos de CVRS.

\begin{tabular}{lllllll}
\hline SF-36v2 & $\begin{array}{l}\text { COOP/ } \\
\text { WONCA }\end{array}$ & NHP & EQ-5D & SPITZER & $\begin{array}{l}\text { WHOQOL- } \\
\text { BREF }\end{array}$ & $\begin{array}{l}\text { QWB- } \\
\text { SA }\end{array}$ \\
\hline
\end{tabular}

1.1. Nivel oracional

\begin{tabular}{lllllll}
\hline (a) Plano $\quad+/-$ & $+/-$ & $+/-$ & $+/-$ & - & - & -
\end{tabular}
léxico-

semántico

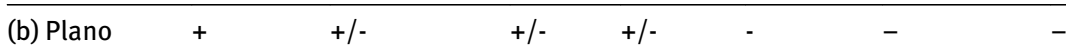
morfosintácti-

co

\begin{tabular}{llllllll}
\hline $\begin{array}{l}\text { (c) Plano } \\
\text { ortográfico }\end{array}$ & $+/-$ & $+/$ & - & - & - & - & - \\
\hline
\end{tabular}

1.2. Nivel supraoracional

\begin{tabular}{lllllll}
\hline (a) Plano & + & + & + & $+/-$ & $+/-$ & $+/-$
\end{tabular}
textual

2.1 Nivel conocimiento mundo

\begin{tabular}{lllllllll}
\hline $\begin{array}{l}\text { (a) Plano } \\
\text { conceptual }\end{array}$ & ++ & $+/-$ & & + & $(+)$ & $+/-$ & $(+)$ & - \\
\hline
\end{tabular}

Interpretación:

++ muy bien (MB)

$+\quad$ bien/muy bien $(\mathrm{B} / \mathrm{MB})$

(+) regular/bien $(\mathrm{R} / \mathrm{B})$

$+/-\operatorname{regular}(\mathrm{R})$

- deficiente (D)

- $\quad$ muy deficiente (MD)

A partir de lo expuesto, consideramos preciso insistir en las equivalencias lingüística y sociopragmática. Las cuestiones lingüísticas deben tenerse más en cuenta. Hay traducciones poco familiarizadas con el código gramatical y con las normas de estilo del español, así como equívocos y desaciertos lingüísticos que no hacen sino enturbiar la comprensión del texto. Los traductores deben tender hacia el orden temático más propio de cada lengua en detrimento del enunciado inglés ya que el objetivo fundamental de la traducción de textos no es otro que reproducir, de la forma más atinada y natural, el mensaje de la lengua de partida con la equivalencia semántica, estilística y discursiva de la lengua de llegada.

El contexto cultural en el que se enmarca el cuestionario se debe tener presente, para lo que el traductor debe poseer, además del cabal conocimiento de las lenguas, una considerable cultura. 
La figura del lingüista como revisor y corrector de estilo es necesaria en la última parte de la cadena, tanto para la corrección desde el punto de vista léxicosemántico, morfosintáctico y ortográfico como para la revisión final de la versión definitiva. Es necesario un verdadero conocedor de la lengua a la que se traduce para que compruebe si el resultado obtenido es adecuado y natural.

Dada la enorme confusión en la que caen los autores al referirse a los tipos de equivalencia durante el proceso de traducción, es conveniente que establezcamos de forma clara qué aspectos consideramos en cada una de las mismas. La falta de claridad, el solapamiento y la ausencia de uniformidad terminológica es una constante en este campo, por lo que urge estandarizar la terminología de los tipos de equivalencia.

Nuestras observaciones, reflexiones y recomendaciones han sido formuladas en la confianza de que las soluciones que ofrecemos puedan contribuir a una mejora en la calidad de las traducciones.

\section{Bibliografía}

Acquadro, Catherine, et al: “Language and Translation Issues." Quality of Life and Pharmacoeconomics in Clinical Trials. Ed. Bert Spilker, Philadelphia: Lippincott-Raven Publishers, 1996:575-585.

Acquadro, Catherine: "ERIQA Recommendations for Translation and Cultural Adaptation of HRQL Measures." ISPOR 6th Annual European Congress. Barcelona, España. Nov. 2003:9-11.

Acquadro, Catherine et al: "Literature Review of Methods to Translate Health-Related Quality of Life Questionnaires for Use in Multinational Clinical Trials." Value in Health 11.3 (2008):509-521.

Acquadro, Catherine et al.: Linguistic Validation Manual for Patient-Reported Outcomes (PRO) Instruments. Lyon: Mapi Research Institute, 2012.

Alonso Caballero, Jordi y Josep Maria Antó Boqué: “Instrumentos de Medida de la Calidad de Vida Relacionada con la Salud: Características Generales y Proceso de Adaptación Transcultural." Quaderns CAPS 14 (1990):16-24.

Alonso Caballero, Jordi et al: "Spanish Version of the Nottingham Health Profile: Translation and Preliminary Validity." American Journal of Public Health 80 (1990):704-708.

Alonso Caballero, Jordi et al. "The Spanish version of the Nottingham Health Profile: a review of adaptation and instrument characteristics." Quality of Life Research 3 (1994):385-393.

Alonso Caballero, Jordi et al: "La Versión Española del SF-36 Health Survey (Cuestionario de Salud SF-36): Un Instrumento para la Medida de los Resultados Clínicos.” Medicina Clínica 104 (1995):771-776.

Alonso Caballero, Jordi: "La Medida de la Calidad de Vida Relacionada con la Salud en la Investigación y la Práctica Clínica.” Gac Sanit 14, 2 (2000):163-167.

Anderson, Robert T. et al.: "International Use and Application of Generic Health-Related Quality of Life Instruments." Quality of Life and Pharmacoeconomics in Clinical Trials. Ed. Bert Spilker, Philadelphia: Lippincott-Raven Publishers, 1996:613-632. 
Badia, Xavier: "Sobre la Adaptación Transcultural de Medidas de la Calidad de Vida Relacionada con la Salud para su Uso en España.” Medicina Clínica 105 (1995):56-58.

Badia, Xavier et al.: “La Versión Española del EuroQol: Descripción y Aplicaciones.” Medicina Clínica, 112 (Supl 1) (1999):79-86.

Badia, Xavier et al.: La Medida de la Salud, 3ª ed., Barcelona: Edimac, 2002.

Beaton, Dorcas et al.: "Guidelines for the Process of Cross-Cultural Adaptation of Self-Report Measures.” Spine 25, 24 (2000):3186-3191.

Beck, Cheryl T. et al.: “Methods to Document Semantic Equivalence of a Translated Scale." Research in Nursing and Health, 26 (2003):64-73.

Behling, Orlando, and Kenneth S. Law, eds.: Translating questionnaires and other research instruments: Problems and solutions. Vol. 133. Thousand Oaks, CA: Sage Publications, 2000.

Bowden, Annabel, and Julia A. Fox-Rushby: "A systematic and critical review of the process of translation and adaptation of generic health-related quality of life measures in Africa, Asia, Eastern Europe, the Middle East, South America." Social Science \& Medicine 57.7 (2003):1289-1306.

Brislin, Richard W.: "Back-translation for cross-cultural research." Journal of cross-cultural psychology 1.3 (1970):185-216.

Brislin, Richard W.: "Translation issues: Multi-language Versions and Writing Translatable English." Proceedings of the Annual Convention of the American Psychological Association. American Psychological Association, 1972.

Brislin, Richard W.: "Questionnaire Wording and Translation." Cross-Cultural Research Methods. Eds. Richard Brislin et al. New York: John Wiley and Sons, 1973:32-58.

Brislin, Richard W.: “Comparative Research Methodology: Cross-Cultural Studies.” International Journal of Psychology 11.3 (1976):215-229.

Brislin, Richard W.: "The Wording and Translation of Research Instruments." Field methods in cross-cultural psychology. Eds. Walter J. Lonner, and John W. Berry. Newbury Park, CA: Sage Publications, 1986:137-164.

Bullinger, Monika et al.: “Translating Health Status Questionnaires and Evaluating their Quality: The IQOLA Project Approach.” J Clin Epidemiol 51.11 (1998):913-923.

Cella, David et al.: "Spanish Language Translation and Initial Validation of the Functional Assessment of Cancer Therapy Quality of Life Instrument.” Medical Care 36.9 (1998): 1407-1418.

Conway, Katrin, and Catherine Acquadro.: "Development of a Checklist to Assess the Quality of Translations of Patient-Reported Outcomes (PRO) Instruments." Value in Health 12.3 (2009): A31.

Congost-Maestre, Nereida: “Adaptación Intercultural de Cuestionarios de Calidad de Vida Relacionada con la Salud: Análisis Contrastivo Inglés-Español de la Dimensión de Función Física en el QWB-SA y el SF-36v2." Actas del I Congreso Internacional sobre Lenguaje y Asistencia Sanitaria [CD-ROM]. Alicante: Instituto IULMA 24-26 Oct. 2007.

Congost-Maestre, Nereida: "Aspectos Socioculturales en la Traducción de Cuestionarios de Salud Estadounidenses.” Panace@ 13 (35) (2012):91-98.

Corless, Inge B et al.: “Issues in Cross-Cultural Quality of Life Research.” Journal of Nursing Scholarship 33.1 (2001):15-20.

Erkut, Sumru: "Developing Multiple Language Versions of Instruments for Intercultural Research." Child development perspectives 4.1 (2010):19-24.

Esteve-Vives, Joaquim et al.: “Adaptación Transcultural de un Cuestionario de Salud.” Rev Esp Reumatol 18 (1991):254-257. 
Esteve-Vives, Joaquim et al.: "Spanish Version of the Health Assessment Questionnaire: Reliability, Validity and Transcultural Equivalency.” The Journal of Rheumatology 20 (1993): 2116-22.

Falcão, Dircilene da Mota et al.: "Translation and Cultural Adaptation of Quality of Life Questionnaires: An Evaluation of Methodology." Journal of Rheumatology 30. 2 (2003):379-385.

Gaite, Luis et al.: "Traducción y Adaptación Transcultural de Instrumentos de Evaluación en Psiquiatría: Aspectos Metodológicos.” Archivos de Neurobiología 60.2 (1997):91-111.

Guarnaccia, Peter J.: "Anthropological Perspectives: The Importance of Culture in the Assessment of Quality of Life." Quality of Life and Pharmacoeconomics in Clinical Trials ( $2^{\text {nd }}$ ed.). Ed. Bert Spilker, Philadelphia: Lippincott-Raven Publishers, 1996:523-528.

Guillemin, Francis et al.: "Cross-Cultural Adaptation of Health-Related Quality of Life Measures: Literature Review and Proposed Guidelines.” J Clin Epidemiol 46 (1993):1417-1432.

Guyatt, Gordon H.: "The Philosophy of Health-Related Quality of Life Translation.” The International Assessment of Health-Related Quality of Life. Theory, Translation, Measurement and Analysis. Eds. Sally Shumaker and Richard Berzon, Oxford: Rapid Communications, 1995:139-143.

Herdman, Michael et al.: “'Equivalence' and the Translation and Adaptation of Health-Related Quality of Life Questionnaires." Quality of Life Research 6 (1997):237-247.

Herdman, Michael et al.: "A Model of Equivalence in the Cultural Adaptation of HRQOL Instruments: The Universalist Approach.” Quality of Life Research 7 (1998):323-335.

Herdman, Michael et al.: "Producing Other Language Versions of the EQ-5D." The Measurement and Valuation of Health Status Using EQ-5D: A European Perspective. Eds. Richard Brooks et al. The Netherlands: Kluwer Academic Publishers, 2003:183-189.

Hilton, Ann, and Myriam Skrutkowski: "Translating Instruments into Other Languages: Development and Testing Proceses." Cancer Nursing 25. 1(2002):1-7.

Hunt, Sonja: “Cross-Cultural Issues in the Use of Socio-Medical Indicators." Health Policy 6 (1986):149-158.

Hunt, Sonja: “Cross-Cultural Comparability of Quality of Life Measures.” Drug Information Journal 27 (1993):395-400.

Hunt, Sonja et al: "Cross-Cultural Adaptation of Health Measures." Health Policy 19 (1991):33-44. Hunt, Sonja, and Stephen McKenna: "Cross-Cultural Comparability of Quality of Life Measures." British Journal of Medical Economics 4 (1992):17-23.

Jones, Patricia et al.: “An Adaptation of Brislin's Translation Model for Cross-Cultural Research." Nursing Research 50.5 (2001):300-304.

Knudsen, Helle Charlotte et al.: "Translation and Cross-Cultural Adaptation of Outcome Measurements for Schizophrenia." British Journal of Psychiatry 177 (suppl. 39) (2000):8-14.

Leplège, Alain, and Angelo Verdier: "The Adaptation of Health Status Measures: Methodological Aspects of the Translation Procedure." The International Assessment of Health-Related Quality of Life. Theory, Translation, Measurement and Analysis. Eds. Sally Shumaker and Richard Berzon, Oxford: Rapid Communications, 1995:93-101.

Lizán Tudela, Luis y Abilio Reig Ferrer: "Adaptación Transcultural de una Medida de la Calidad de Vida Relacionada con la Salud: La Versión Española de las Viñetas COOP/WONCA.” Atención Primaria 24. 2 (1999):75-82.

Massoubre, Catherine et al.: "La Traduction des Questionnaires et des Tests: Techniques et Problèmes.” Canadian Journal of Psychiatry / Rev Can Psychiatre 47 (2002):61-67.

Matías-Carrelo, Leida E. et al.: "The Spanish Translation and Cultural Adaptation of Five Mental Health Outcome Measures.” Culture, Medicine and Psychiatry 27.3 (2003):291-313. 
Patrick, Donald L. et al.: “Cross-Cultural Validation of Quality of Life Measures.” Quality of Life Assessment: International Perspectives. Eds. John Orley and Willem Kuyken. Berlin: Springer Verlag, 1994:19-32.

Sánchez-Reyes, María Sonsoles y Manuel Martín Casado: “Más allá de la Traducción: La Validación de Cuestionarios Científico-Técnicos Inglés-Español.” Livius (Revista de Estudios de Traducción) 14 (1999):149-155.

Sartorius, Norman, and Willem Kuyken: "Translation of health status instruments". Quality of Life Assessment: International Perspectives. Eds. John Orley and Willem Kuyken. Berlin: Springer Verlag, 1994:3-18.

Scientific Advisory Committee of the Medical Outcomes Trust: "Assessing Health Status and Quality of Life Instruments: Attributes and Review Criteria." Quality of Life Research 11 (2002):193-205.

Sechrest, Lee et al.: "Problems of Translation in Cross-Cultural Research." Journal of CrossCultural Psychology 3. 1 (1972):41-56.

Serra-Sutton, Vicky i Michael Herdman: “Metodología d'Adaptació Transcultural d’Instruments de Mesura de la Qualitat de Vida Relacionada amb la Salut.” Informatiu AATM 24 (2001): 18-20.

Skevington, Suzanne: “Advancing Cross-Cultural Research on Quality of Life: Observations Drawn from the WHOQOL Development." Quality of Life Research 11 (2002):135-144.

Sousa, Valmi, and Wilaiporn Rojjanasrirat: "Translation, Adaptation and Validation of Instruments or Scales for Use in Cross-Cultural Health Care Research: A Clear and User-Friendly Guideline." Journal of Evaluation in Clinical Practice 17 (2011):268-274.

Valderas, José María et al.: "Instrumentos de Medida de Calidad de Vida Relacionada con la Salud y de Otros Resultados Percibidos por los Pacientes." Med Clin 125.1(2005):56-60.

van Widenfelt, Brigit M. et al.: "Translation and Cross-Cultural Adaptation of Assessment Instruments Used in Psychological Research with Children and Families." Clinical Child and Family Psychology Review 8. 2(2005):135-147.

Vázquez-Ayora, Gerardo: Introducción a la Traductología. Washington: Georgetown University Press, 1977.

Wagner, Anita et al.: "Cross-cultural Comparisons of the Content of SF-36 Translations across 10 Countries: Results from the IQOLA Project." J Clin Epidemiol 51.11(1998):925-932.

Wild, Diane et al.: "Principles of Good Practice for the Translation and Cultural Adaptation Process for Patient-Reported Outcomes (PRO) Measures: Report of the ISPOR Task Force for Translation and Cultural Adaptation." Value in Health (The Official Journal of the ISPOR) 8. 2 (2005):94-104. 


\section{ANEXO}

\section{SF-36v2}

\section{Cuestiones lingüisticas}

\subsection{Nivel oracional}

(a) Plano léxico-semántico - Palabra mal traducida en el contexto (preguntas).

- Modificaciones (sustituciones, inclusiones y omisiones) adecuadas.

- Redundancia léxica (y sintáctica) en instrucciones, pregunta 3 y respuestas a preguntas 2 y 5 .

- Grado de formalidad alto (limitar).

- Uso de la palabra moderado.

\section{(b) Plano morfosintáctico - Bueno.}

(c) Plano ortográfico - Puntuación insuficiente o inadecuada (por favor lleva coma, solo adjetivo no lleva tilde, fácilmente lleva acento, uso incorrecto de puntos suspensivos).

\begin{tabular}{ll}
\hline 1.2. Nivel supraoracional & - Organización textual buena. \\
(plano textual) & - Uso de fórmulas corteses. \\
& - No hay adecuación de género.
\end{tabular}

\section{Cuestiones socioculturales}

2.1 Nivel sociopragmático - La forma de marcar el cuestionario es apropiada (plano conceptual) (mark an X/marque con una X).

- Adaptación positiva a nuestro contexto de la palabra groups, de la expresión problema emocional, de deportes, actividades, tareas domésticas y distancias.

\section{Calificación: MB}

Comentario: Es un cuestionario modelo, aunque debe revisar algunos aspectos lingüísticos, sobre todo en los planos léxico-semántico y ortográfico.

\section{COOP/WONCA}

\section{Cuestiones lingüísticas}

\subsection{Nivel oracional}

\begin{tabular}{ll}
\hline (a) Plano léxico- & - Redundancia léxica en preguntas y respuestas (viñetas 3 y 7). \\
semántico & - Grado formalidad alto (emocional, limitar, calificar). \\
& - Repetición léxica de la palabra moderado y moderadamente. \\
& - Oración larga con muchos conceptos (viñeta 3).
\end{tabular}




\section{Cuestiones lingüísticas}

\subsection{Nivel oracional}

(b) Plano morfosintáctico - Conjunciones que producen confusión (viñeta 2).

- Uso de adverbios formales acabados en -mente.

(c) Plano ortográfico - Puntuación inadecuada (puntos suspensivos).

1.2. Nivel supraoracional - Organización textual mejorada en castellano con instrucciones y (plano textual) uso de fórmulas corteses.

- No necesita adecuación de género.

\section{Cuestiones socioculturales}

2.1 Nivel pragmático (plano conceptual)
- La forma de marcar el cuestionario no se adapta, es adecuada en el formato (rodee con un círculo el número).

- El concepto de problema emocional se soluciona con ejemplos en original (viñeta 2).

- La adaptación del concepto grupos se ha de considerar (viñeta 4).

Calificación: B/MB

Comentarios: Tiene que revisar aspectos lingüísticos y cuestión pragmática.

\section{NHP}

\section{Cuestiones lingüisticas}

\subsection{Nivel oracional}

\begin{tabular}{ll}
\hline (a) Plano léxico- & - Palabra mal traducida en el contexto (preguntas). \\
semántico & - Modificaciones (inclusiones y omisiones) sin pérdida semántica en \\
& general. \\
& - Redundancia léxica (y sintáctica) en instrucciones y en todos los \\
& ítems de la segunda parte.
\end{tabular}

(b) Plano morfosintáctico - Frases negativas con respuesta sí/no (en proceso de cambio).

- Uso de adverbios formales en -mente.

(c) Plano ortográfico - Puntuación insuficiente o inadecuada (ítems sin punto final, etc. con puntos suspensivos, navidades sin inicial mayúscula).

1.2. Nivel supraoracional - Organización textual muy buena.- Uso de fórmulas corteses. (plano textual) - Hay adecuación de género.

\section{Cuestiones socioculturales}

\begin{tabular}{ll}
\hline 2.1 Nivel pragmático & - La forma de marcar el cuestionario se adapta (put a tick/marcar una \\
(plano conceptual) & cruz). \\
& - Adaptación positiva a nuestro contexto de tareas domésticas, \\
& actividades de ocio y vacaciones.
\end{tabular}

\section{Calificación: B}

Comentario: Tiene que mejorar aspectos lingüísticos. 


\section{EQ-5D}

\section{Cuestiones lingüísticas}

1.1. Nivel oracional

(a) Plano léxicosemántico

- Palabra mal traducida en el contexto (casillero).

- Sustitución adecuada.

- Redundancia léxica (y sintáctica) en instrucciones segunda parte.

- Uso de la palabra moderado y moderadamente.

(b) Plano morfosintáctico - Problema de concordancia nominal y gramatical en instrucciones segunda parte.

(c) Plano ortográfico - Puntuación insuficiente o inadecuada (ítems sin punto final, abreviatura sin punto).

1.2. Nivel supraoracional - Organización textual buena.

(plano textual) - Uso de fórmulas corteses.

- No hay adecuación de género.

\section{Cuestiones socioculturales}

2.1 Nivel pragmático (plano conceptual)
- La forma de marcar el cuestionario se adapta (place a tick/marcar con una cruz).

- No hay otras diferencias culturales destacadas en nuestro contexto.

Calificación: R+

Comentarios: Tiene que mejorar aspectos lingüísticos. Redacción pobre en castellano.

\section{SPITZER'S QLI}

\section{Cuestiones lingüísticas}

\subsection{Nivel oracional}

(a) Plano léxicosemántico
- Uso de expresiones coloquiales (al cien por cien, fatal) como en original.

- Grado de formalidad más alto que en original (aspa, remunerada, significativa, limitado, confinado).

- Oraciones largas con muchos conceptos.

(b) Plano morfosintáctico - Uso excesivo de construcciones gerundivas en castellano.

- Uso excesivo de la conjunción disyuntiva «o».

- Uso de adverbios formales en -mente.

(c) Plano ortográfico - Puntuación insuficiente o inadecuada (por favor lleva coma, sólo adverbio lleva acento, los pronombres interrogativos qué y cómo llevan acento). 


\section{Cuestiones lingüísticas}

\subsection{Nivel oracional}

1.2. Nivel supraoracional - Organización textual mejorada en instrucciones con fórmula cortés (plano textual) en castellano.

- No hay adecuación de género.

\section{Cuestiones socioculturales}

$\begin{array}{ll}\text { 2.1 Nivel pragmático } & \text { - La forma de marcar el cuestionario se adapta (put a circle/ponga un } \\ \text { (plano conceptual) } & \text { aspa). } \\ & \text { - La adaptación del concepto de actividades voluntarias y el de } \\ & \text { institución se ha de considerar. }\end{array}$

\section{Cuestiones visuales}

3.1 Nivel técnico-
semiótico (plano visual)

Calificación: R

Comentario: Tiene que revisar y mejorar aspectos lingüísticos y visuales.

\section{WHOQOL-BREF}

\section{Cuestiones lingüisticas}

\subsection{Nivel oracional}

\begin{tabular}{|c|c|}
\hline $\begin{array}{l}\text { (a) Plano léxico- } \\
\text { semántico }\end{array}$ & $\begin{array}{l}\text { - Palabras y expresiones mal traducidas (poor- poco, how satisfied- } \\
\text { cúan satisfecho, how available- qué disponible tiene). } \\
\text { - Falsos amigos (ability) } \\
\text { - Modificaciones por sustitución, inclusión u omisión sin cambios } \\
\text { semánticos relevantes. } \\
\text { - Repetición léxica de cuán satisfecho. } \\
\text { - Redundancia léxica en instrucciones. } \\
\text { - Grado de formalidad alto (expectativas, obtener apoyo, } \\
\text { experimenta, ciertos). } \\
\text { - Uso de la palabra moderado. } \\
\text { - Problemas en la escala de gradación de la mayor parte de las } \\
\text { respuestas. }\end{array}$ \\
\hline ) Plano morfo & $\begin{array}{l}\text { - Anglicismo sintáctico en pregunta de datos sociodemográficos } \\
\text { (enfermedad/problema). } \\
\text { - Falta de concordancia temporal entre enunciados e ítems. } \\
\text { - Uso de adverbios formales en - mente. } \\
\text { - Construcción inadecuada con el gerundio (thinking/pensando). } \\
\text { - Omisión de la conjunción «o» en listado de sentimientos negativos } \\
\text { (ítem 26). }\end{array}$ \\
\hline
\end{tabular}




\section{Cuestiones lingüísticas}

1.1. Nivel oracional

(c) Plano ortográfico - Puntuación insuficiente o inadecuada (comas innecesarias, faltan dos puntos en los cuatro enunciados generales, falta signo de interrogación inicial en la última pregunta).

\begin{tabular}{ll}
\hline 1.2. Nivel supraoracional & - Organización textual buena. \\
(plano textual) & - Uso de fórmulas corteses. \\
& - No hay adecuación de género.
\end{tabular}

\section{Cuestiones socioculturales}

\begin{tabular}{ll}
\hline 2.1 Nivel pragmático & - La forma de marcar el cuestionario no se adapta, es adecuada en el \\
(plano conceptual) & formato (circle the number/haga un círculo). \\
& - No hay otras diferencias culturales destacadas en nuestro \\
& contexto.
\end{tabular}

\section{Calificación: D}

\section{Comentario:}

Plantea problemas léxicos y semánticos en enunciados, preguntas y opciones de respuestas. Redacción pobre en instrucciones e ítems.

Tiene que revisarse el formato (en las dos versiones).

\section{QWB-SA}

\section{Cuestiones lingüísticas}

\subsection{Nivel oracional}

\begin{tabular}{ll}
\hline (a) Plano léxico- & - Palabras o expresiones incoherentes por traducciones literales \\
semántico & (marcas de chequear, boligrafos de felpa, aparatos de salud, dolor \\
& de diente, respiración corta, estómago indispuesto, calambre en el \\
& área pélvica, queja de salud, Islas Pacíficas, etc.). \\
- & Modificaciones por sustitución, inclusión u omisión con pérdida \\
& semántica. \\
- & Falsos amigos (severe, apply, floater). \\
- La palabra polisémica discharge no siempre está bien traducida en \\
el contexto. \\
- Redundancia léxica (y sintáctica) (enunciado 2). \\
- Grado de formalidad alto (escoliosis, visión distorsionada, dolor \\
abdominal, abdomen, defecar, limitación, limitado, emocional). \\
- Oraciones largas con muchos conceptos que dificultan la \\
interpretación. \\
- Registro léxico diferente (principalmente mexicano) (manejar, \\
ardor, comezón, salpullido, anteojos, relumbrón, quijada, \\
coyuntura, cruda, banqueta, etc.).
\end{tabular}




\section{Cuestiones lingüísticas}

\subsection{Nivel oracional}

(b) Plano morfosintáctico - Anglicismos léxico-sintácticos numerosos (no incluyendo, no control, ¿ha tenido Ud. sentir?, ¿tiene falta o parálisis de las manos?, ¿tuvo dificultad para oír, flujo o sangrar de un oído?, ¿tuvo Ud. dolor o estar sangrando de los labios?, etc.)

- Problema de concordancia gramatical de género y número (escoliasis severo, estado de salud buena).

- Modificaciones de tiempos verbales, modos y voces inadecuadas.

- Exceso de conjunciones disyuntivas «o» en las oraciones.

- Omisión de conjunciones o uso incorrecto de las mismas.

- Omisión de artículos determinados e indeterminados delante de sustantivos.

- Omisión de preposiciones o uso incorrecto de las mismas.

(c) Plano ortográfico - Puntuación insuficiente o inadecuada (por favor lleva coma, exceso de comas, faltan signos de interrogación al comienzo de las frases, faltan acentos, uso incorrecto de puntos suspensivos, etc.).

1.2. Nivel supraoracional - Organización textual pobre.

(plano textual) - No incluye las cabeceras de las cinco secciones del original.

- No hay adecuación de género.

- Mantiene fórmula cortés al principio pero suprime la de agradecimiento final.

\section{Cuestiones socioculturales}

\subsection{Nivel pragmático} (plano conceptual)
- La forma de marcar el cuestionario no se adapta (fill in the circle/ rellenar el círculo). No es un procedimiento usual en nuestro país pero es adecuada en el formato.

- No se adaptan conceptos tales como tylenol, Medi-van (camioneta médica) o la relación de tipos de estudios.

Calificación: D/MD en nuestro contexto.

Comentario: Se recomienda una revisión completa del cuestionario en todos los aspectos:

- Plantea problemas léxicos, sintácticos y semánticos.

- Plantea problemas de registro léxico al haber una única versión para todos los países de habla hispana.

- Tiene que revisar la organización textual, adaptar las diferencias culturales y prestar atención al formato. 